Document downloaded from:

http://hdl.handle.net/10251/167117

This paper must be cited as:

Bellido, P.; Seimetz, M.; Soriano, A.; Huertas, C.; García Lopez, J.; Jimenez-Ramos, MC.; Fernandez, B.... (2013). Dosimetric Calibration of Radiochromic Film For Laser-accelerated Proton Beams. IEEE. 20-23. https://doi.org/10.1109/NSSMIC.2013.6829806

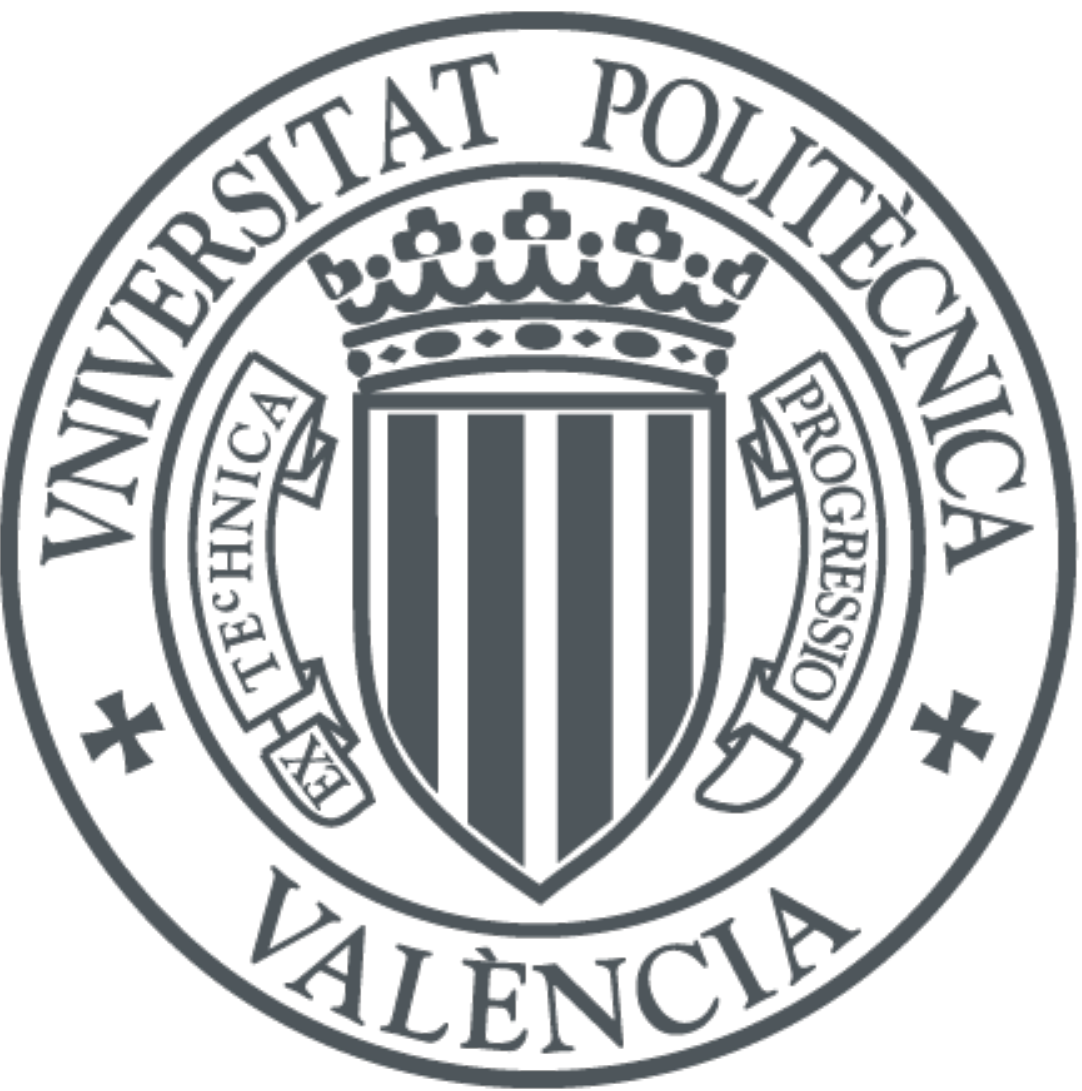

The final publication is available at

https://doi.org/10.1109/NSSMIC.2013.6829806

Copyright IEEE

Additional Information 


\title{
Dosimetric Calibration of Radiochromic Film For Laser-accelerated Proton Beams
}

\author{
P. Bellido*, M. Seimetz, A. Soriano, C. Huertas, J. García López, M. C. Jiménez-Ramos, B. Fernández, P. Conde, \\ E. Crespo, A. J. González, L. Hernández, A. Iborra, F. Martos, L. Moliner, J. P. Rigla, M. J. Rodríguez-Álvarez, \\ F. Sánchez, L. F. Vidal, and J. M. Benlloch
}

\begin{abstract}
When an ultra-intense and ultra-short laser pulse interacts with solid matter a fraction of the laser pulse can be converted into kinetic energy of a beam of charged particles. Radiochromic film (RCF), widely used as radiation detector in the field of conventional radiotherapy, can be used as detector for laser-accelerated protons. If used in stack configuration it is a useful and versatile tool to obtain $2 \mathrm{D}$ spatial distribution and energetic information of proton beams.

In order to obtain dosimetric information from RCF it must be properly calibrated. Irradiating film pieces under well known conditions allows us to establish a relation between the optical density (OD) of the radiochromic film, which is measured through a flat bed scanner operating in transmission mode, and the deposited energy in the active layer. A calibration curve over a large dynamic range ( 3 orders of magnitude) has been obtained for few $\mathrm{MeV}$ protons. Our calibration process has been performed at the Spanish National Accelerator Center at Sevilla. We have irradiated several areas of a single RCF with a constant $50 \mathrm{pA}$ beam current and fixed $4 \mathrm{MeV}$ energy from a $3 \mathrm{MV}$ tandem accelerator. We have calculated the deposited energy in the films under the same conditions. We demonstrate that this technique can be used to measure the spectrum and total energy of a laser-accelerated mixed-energy proton beam.

This detector has been calibrated for a near future application at the Center of Pulsed, Ultra-short, Ultra-intense Lasers (CLPU) at Salamanca (Spain).

We present the calibration procedure and results, the design optimization, and a comparison with similar experiments.
\end{abstract}

\section{INTRODUCTION}

$\mathbf{I}$ $\mathrm{N}$ a typical experiment of laser-proton acceleration [1], an ultra-intense and ultrashort laser pulse interacts with a thin target of metal or other materials. The plasma created in the interaction results in the acceleration of protons towards the target normal direction. Generally, a broad proton spectrum

Manuscript received November 11, 2013. Project funded by the Spanish Ministry of Economy and Competitiveness and co-funded with FEDER's funds within the INNPACTO 2011 program. This work was supported by the Spanish Plan Nacional de Investigación Científica, Desarrollo e Innovación Tecnológica (I+D+i) under Grant No. FIS2010-21216-CO2-01 and the Valencian Local Government under Grants PROMETEOII/2013/010 and ISIC 2011/013.

P. Bellido, M. Seimetz, A. Soriano, C. Huertas, P. Conde, E. Crespo, A. J. González, L. Hernández, A. Iborra, F. Martos, L. Moliner, J. P. Rigla, M. J. Rodríguez-Álvarez, F. Sánchez, L. F. Vidal, and J. M. Benlloch are with Instituto de Instrumentación para Imagen Molecular (I3M), CSIC Universidad Politécnica de Valencia - CIEMAT, Camino de Vera s/n, Ed. 8-N-1, 46022 Valencia, Spain.

J. García López, M. C. Jiménez-Ramos, and B. Fernández are with Centro Nacional de Aceleradores (CNA), U. Sevilla - J. Andalucía - CSIC, Avda. Thomas Alva Edison 7, 41092 Sevilla, Spain.

*Corresponding author. Contact: pbellido@i3m.upv.es. with a maximum cut off energy is obtained. Many experiment results can be described by an exponential decay,

$$
\frac{d N(E)}{d E}=\frac{N_{0}}{E} \exp \left(-\frac{E}{k_{B} T}\right),
$$

where $N_{0}$ represents the total number of protons and $k_{B} T$ is a mean energy. At laser pulse energies around $1 \mathrm{~J}$ maximum proton energy of 1-10 MeV have been observed.

Radiochromic films provide high-resolution 2D dose distributions with less than $3 \mu \mathrm{m}$ of spatial resolution. For this reason, we have capability to measure single pencil beam interaction with the film and large spots due to broad beams. Thus, we will be able to characterize different beam qualities like beam size, divergence and emittance.

Using the RCFs in stack configuration (Figure 1) an energyresolved measurement is possible. Protons are stopped in each film in function of their initial energy. The range of each proton energy in the stack provides information to deconvolve the spectrum. A procedure to reconstruct the proton energy spectrum using an RCF stack can be found in [2]. Other examples of the use of RCF like a proton detector can be found in [3], [4].

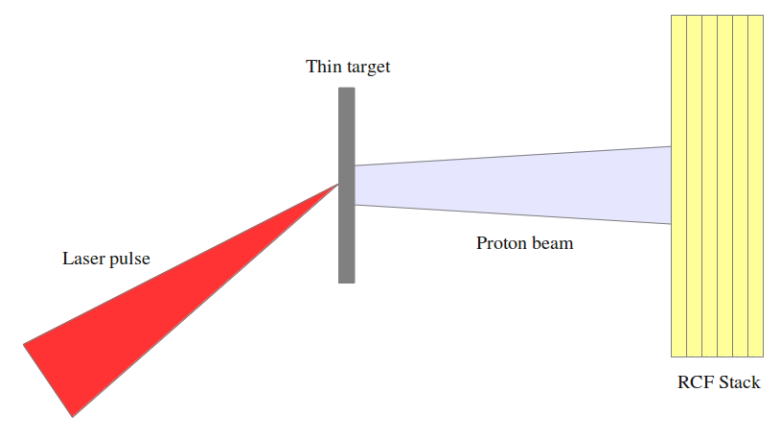

Fig. 1. Schematic experimental setup. RCF stack provides $2 \mathrm{D}$ and depth dosimetric information.

\section{MATERIALS AND METHODS}

Ionizing radiation changes the RCF optical density (OD) as a function of the energy deposited in the sensitive layer. This 


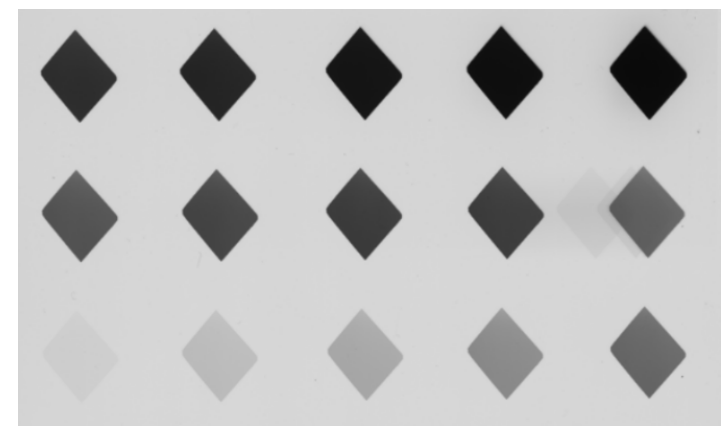

Fig. 2. HD-V2 after several irradiation times at constant beam current. Different doses generate an OD gray spectrum.

amount of energy depends on the number of protons and their interacting stopping power. Based on previous calculations of the expected dose deposition of the accelerated particles we have chosen HD-V2 Gafchromic ${ }^{\mathrm{TM}}$ radiochromic film as detector material. These films are formed by a 12 micron thin active layer and a base of 97 microns of polyester. The active layer is made of a compound such that the impact of radiation leads to a polymerization that changes the absorption spectrum. The films have to be digitized without chemical etching for quantitative data analysis after irradiation. We have carried out this process through the flat bed scanner Epson Perfection ${ }^{\mathrm{TM}}$ V750 PRO, operated in transmission mode, obtaining 48 bit color images of 300 dpi. Selecting a region of interest (ROI), we measure the red channel pixel value $\left(\mathrm{PV}_{\text {red }}\right)$ and this is converted to a relative measure of the RCF optical density $\left(\mathrm{OD}_{n e t}\right)$ in relation with the background value $\left(\mathrm{BV}_{\text {red }}\right)$,

$$
\mathrm{OD}_{n e t}=\log \left(\frac{\mathrm{BV}_{\text {red }}}{\mathrm{PV}_{\text {red }}}\right)
$$

The scanner has passed several acceptance tests such as reproducibility and uniformity of the scan, obtaining results below $1 \%$ deviation.

\section{IRRADIATION}

Irradiating film pieces under well known conditions allows for establishing a relation between the optical density of the RCF and the deposited energy in the active layer. Our calibration process has been performed at the Spanish National Accelerator Center at Sevilla. There, a 3 MV tandem accelerator produces a homogeneous, collimated, and monoenergetic proton beam. $4 \mathrm{MeV}$ fixed energy and $50 \mathrm{pA}$ proton beam current have been selected as calibration parameters. We have irradiated only a single RCF because $4 \mathrm{MeV}$ protons pass completely through the film and the deposited energy is uniform (far from the Bragg peak). The beam size at the RCF position is $80 \mathrm{~mm}^{2}$. Different irradiation times in different areas generate an OD gray spectrum (Figure 2). Irradiation times vary from $1 \mathrm{~s}$ to $1000 \mathrm{~s}$. The total charge delivered in each irradiation time has been measured with a Faraday cup situated behind the RCF.

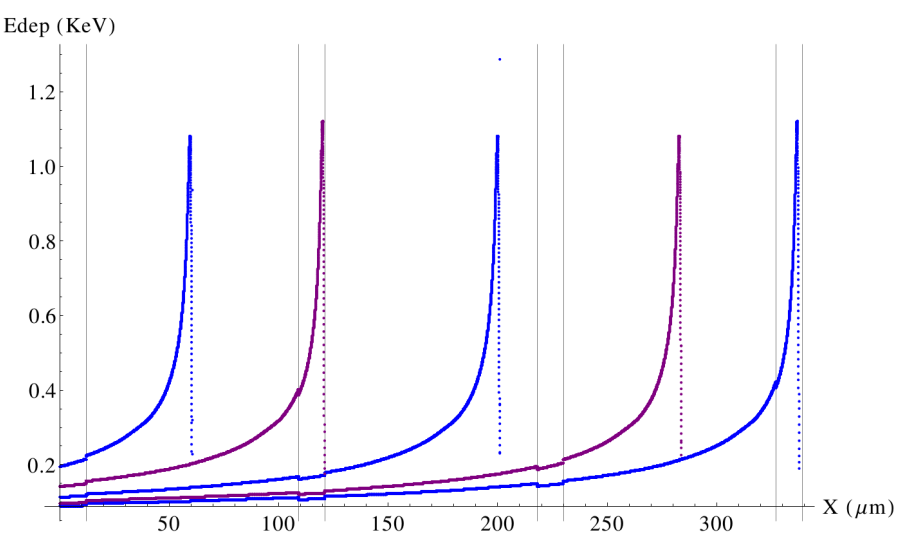

Fig. 3. Calculated depth dose profile for 2, 3, 4, 5 and $5.5 \mathrm{MeV}$ single protons.

\section{Calculation}

Simulations of the interaction of protons with matter are needed to calculate the energy deposited in the films. The SRIM code [5] has been used to calculate stopping power tables in RCF (the chemical composition and densities of the different layers are given by the manufacturer). With this table and a ray-tracing code we obtain a depth dose profile for a single proton that is used to calculate the total energy deposited in the active layer of a single film or of several layers in a stack configuration film, where the protons pass through multiple layers (Figure 3).

We have obtained a calibration curve that relates the net OD with the deposited energy density (Figure 4). The experimental curve has been fitted to a polynomial expression,

$$
\log \left(E_{d}\right)=\sum_{i=0}^{9} a_{i} \mathrm{OD}^{i}
$$

This curve is valid for a large dynamic range $\left(10^{3}\right)$ as stated in [1] and is consistent with results of other authors [2], [6]. Below the dynamic range obtained we cannot perform measurements with this type of film because we cannot differentiate the OD value from the background. On this case more sensitive types like MD-45 or HS may be used. In the opposite situation, above the dynamic range available, we can apply the HD-V2 film placing a filter between the proton beam and the films decreasing the energy of the incident particles. Another option to increase the dynamic range is to use the blue pixel value which for high OD is significantly more sensitive than the red value. It is not possible to obtain a precise calibration curve with the blue channel pixel value for the entire energy range because for low and medium energies, variations of the pixel value are very small. However, beyond our calibration range we have observed more sensitivity for the blue pixel than for the red one (Figure 5).

\section{VALIDITY OF THE CALIBRATION CURVE}

We have performed test irradiations of film stacks in order to check the validity of our method to measure laser-proton distributions. We compare the calculated energy with the 


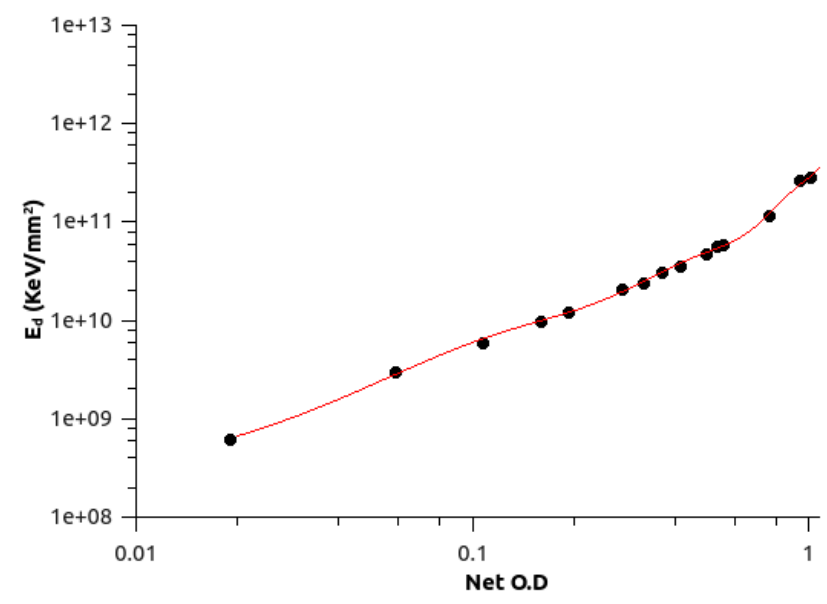

Fig. 4. Double logarithmic plot of the experimental calibration curve fitted with a polynomial.

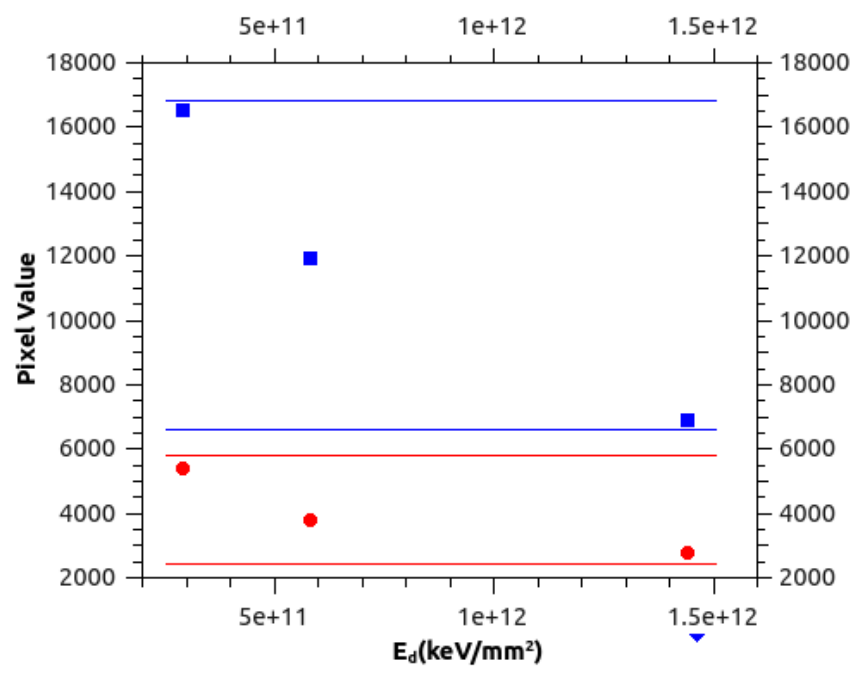

Fig. 5. Experimental measurements of the red and blue pixels in the high energy range. The range of the blue pixel value is larger than the red one, implying increased sensitivity.

measured one. We have to take into account an important detail of the RCF response. These exhibit a $20 \%$ dose suppression for protons near the Bragg peak due to a quenching effect [7]. Appropriate corrections have been carried out.

We have irradiated a 4-film stack with a $5.5 \mathrm{MeV}$ proton energy. We have selected this energy because the Bragg peak coincides with the last active layer (Figure 3). The total charge is $1500 \mathrm{pC}$. The results are shown in Figure 6. We can observe a good agreement between calculated and measured energy. In addition, we have made a multi-energy test. We have irradiated the same 4-film stack with several proton energies $(2.00,3.03$, $4.08,4.98,5.57 \mathrm{MeV})$. The total charge for each beam is 500 pC. The results are show in Figure 7.

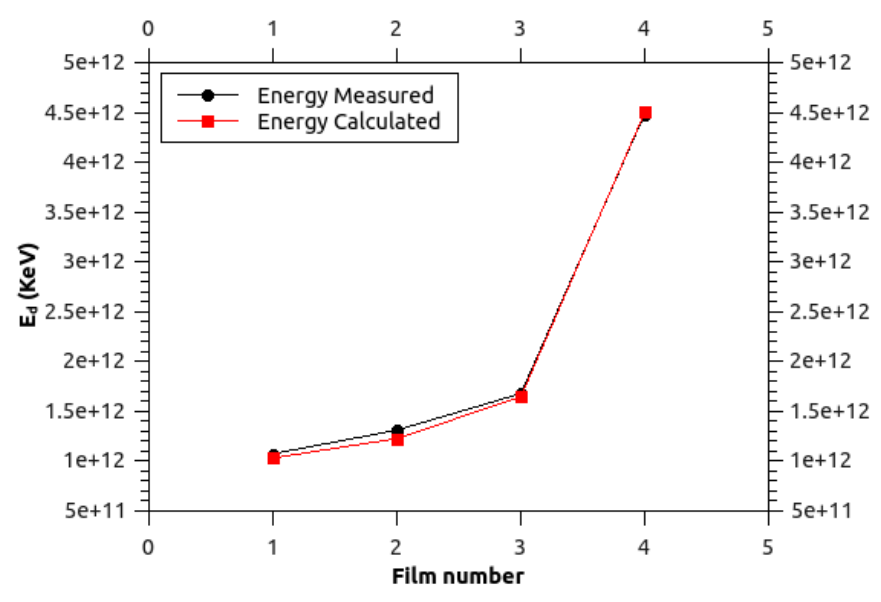

Fig. 6. Four film measurements of the $5.5 \mathrm{MeV}$ depth dose profile.

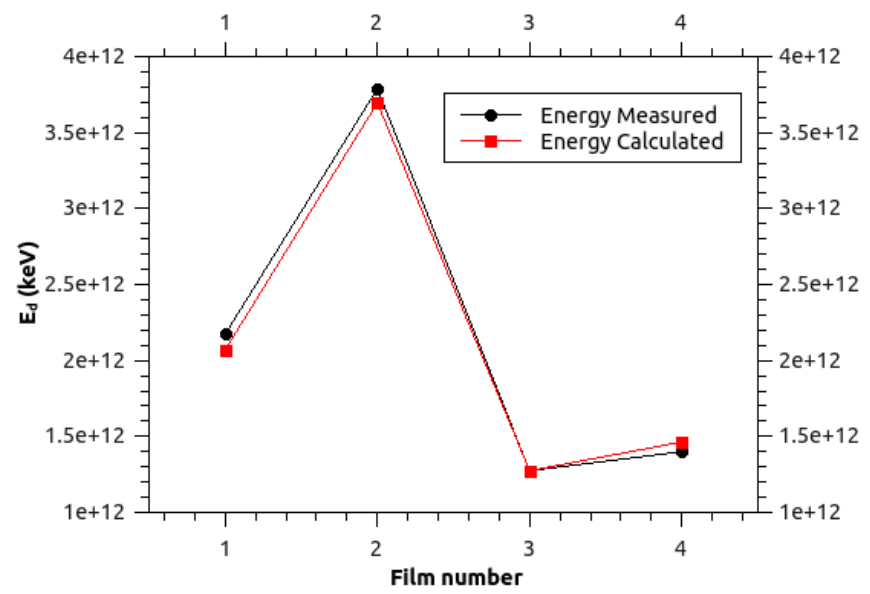

Fig. 7. Energy deposited in each film irradiated by 2.00, 3.03, 4.08, 4.98 and $5.57 \mathrm{MeV}$. Film number 2 is the darkest film due to the large energy deposited by the $3.03 \mathrm{MeV}$ proton beam. The $3.03 \mathrm{MeV}$ proton Bragg peak is located in this layer.

\section{RECONSTRUCTION OF A LASER-PROTON ACCELERATED SPECTRUM}

Once we have a well tested calibration curve that is able to relate the RCF OD with the deposited energy, our main aim is to reconstruct a laser-proton accelerated spectrum.

In order to perform a spectroscopy experiment we need more information than a single RCF provides. RCF becomes darker as more energy is deposited, but it is not possible to distinguish between the energy of the incident particle and the number of incident particles. For this reason we need additional information that we can obtain with a stack configuration setup (Figure 1). We have calculated the energy deposition in a specific active layer over a large range of incident particle energies (Figure 8) and we have constructed a function for each film denominated $E_{l o s s-i}$. With this information and the measured deposited energy we will be able to reconstruct the incident spectrum. The total energy 
deposited in film $i$ by a laser-proton beam can be calculated through:

$$
E_{i}=\int_{0}^{E_{\max }} \frac{d N\left(E^{\prime}\right)}{d E} E_{l o s s-i}\left(E^{\prime}\right) d E^{\prime}
$$

where $E_{l o s s-i}$ are the family of functions calculated previously. With a set of experimental measurements $E_{i}$ and assuming a spectral shape like Equation (1) one will finds the best values of the parameters $N_{0}$ and $k_{B} T$ through a leastsquares fitting algorithm.

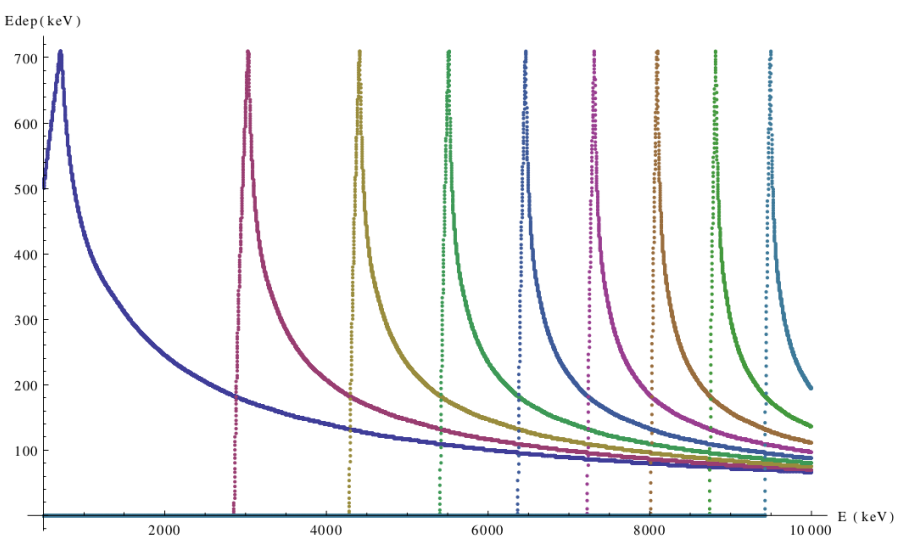

Fig. 8. Each curve (inverse Bragg curve) describes the deposited energy in a specific active layer for a given proton energy.

\section{CONCLUSION}

We have obtained a well tested calibration curve to use $\mathrm{RCF}$ like a multi-MeV proton detector with a large dynamic range of validity. In a stack configuration, we are able to measure the energy deposited in all sensitive layers and obtain 2D dosimetric information, and with this information we will reconstruct the incident proton spectrum.

\section{REFERENCES}

[1] H. Daido, M. Nishiuchi, and A. S. Pirozhkov, "Review of laser-driven ion sources and their applications," Reports on Progress in Physics, vol. 75, no. 5, p. 056401, 2012.

[2] F. Nurnberg, M. Schollmeier, E. Brambrink, A. Blazevic, D. C. Carroll, K. Flippo, D. C. Gautier, M. Geissel, K. Harres, B. M. Hegelich, O. Lundh, K. Markey, P. McKenna, D. Neely, J. Schreiber, and M. Roth, "Radiochromic film imaging spectroscopy of laser-accelerated proton beams," Review of Scientific Instruments, vol. 80, no. 3, p. 033301, 2009.

[3] M. Schollmeier, M. Roth, A. Blazevic, E. Brambrink, J. Cobble, J. Fernandez, K. Flippo, D. Gautier, D. Habs, K. Harres, B. Hegelich, T. Heßling, D. Hoffmann, S. Letzring, F. Nürnberg, G. Schaumann, J. Schreiber, and K. Witte, "Laser ion acceleration with micro-grooved targets," Nuclear Instruments and Methods in Physics Research Section A: Accelerators, Spectrometers, Detectors and Associated Equipment, vol. 577, no. 1-2, pp. 186 - 190, 2007.

[4] P. Antici, S. Gaillard, L. Gremillet, M. Amin, M. Nakatsutsumi, L. Romagnani, M. Tampo, T. Toncian, R. Kodama, P. Audebert, H. Pépin, O. Willi, M. Borghesi, T. Cowan, and J. Fuchs, "Optimization of flat-cone targets for enhanced laser-acceleration of protons," Nuclear Instruments and Methods in Physics Research Section A: Accelerators, Spectrometers, Detectors and Associated Equipment, vol. 620, no. 1, pp. 14 - 17, 2010.

[5] J. Ziegler and J. Biersack, "SRIM - The Stopping and Range of Ions in Solids," Pergamon, 1985. http://www.srim.org/.

[6] D. S. Hey, M. H. Key, A. J. Mackinnon, A. G. MacPhee, P. K. Patel, R. R. Freeman, L. D. V. Woerkom, and C. M. Castaneda, "Use of gafchromic film to diagnose laser generated proton beams," Review of Scientific Instruments, vol. 79, no. 5, p. 053501, 2008.
[7] A. Piermattei, R. Miceli, L. Azario, A. Fidanzio, S. delle Canne, C. De Angelis, S. Onori, M. Pacilio, E. Petetti, L. Raffaele, and M. G. Sabini, "Radiochromic film dosimetry of a low energy proton beam," Medical Physics, vol. 27, no. 7, pp. 1655-1660, 2000. 